EDUCACIÓN Y CRECIMIENTO ECONÓMICO: ANÁLISIS E IMPLICANCIAS

Revista Economía y Administración, Vol. 9, No.1, 2018

\title{
EDUCACIÓN Y CRECIMIENTO ECONÓMICO: ANÁLISIS E IMPLICANCIAS
}

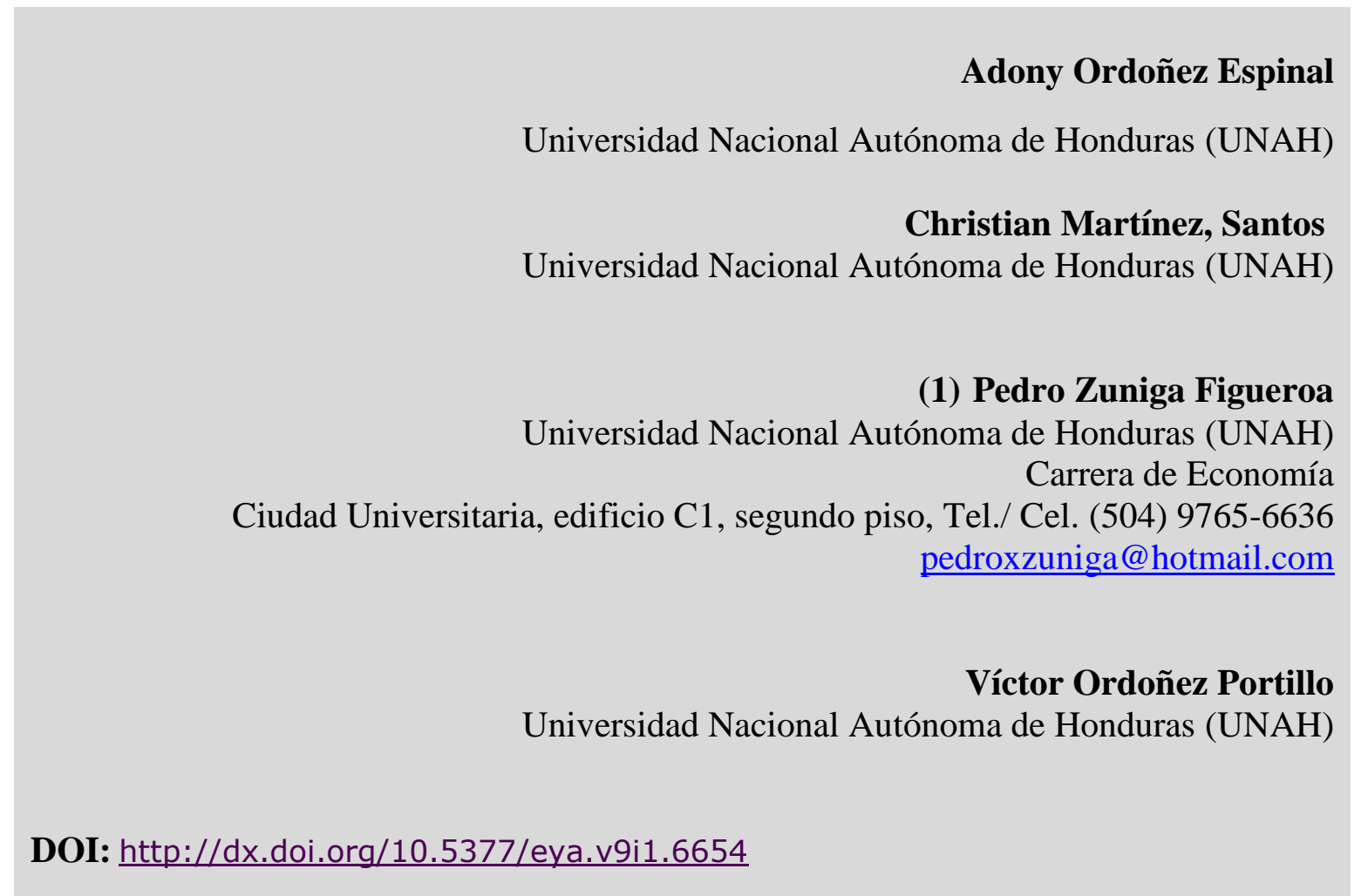

Adony Ordoñez Espinal

Universidad Nacional Autónoma de Honduras (UNAH)

Christian Martínez, Santos

(1) Pedro Zuniga Figueroa

Carrera de Economía

pect./Cel. (504) $9765-6636$

Víctor Ordoñez Portillo

Universidad Nacional Autónoma de Honduras (UNAH)

DOI: http://dx.doi.org/10.5377/eya.v9i1.6654

(1) Autor de contacto

\section{RESUMEN}

Una de las principales causas del desarrollo económico es sin lugar a duda el capital humano, la finalidad de este documento es presentar el vínculo que existe entre la educación y el crecimiento económico y sus diferentes matices, pasando por una perspectiva individual hasta una perspectiva macroeconómica.
Palabras clave: Educación, Gasto Público, Crecimiento Económico. 


\title{
EDUCACIÓN Y CRECIMIENTO ECONÓMICO: ANÁLISIS E IMPLICANCIAS \\ Revista Economía y Administración, Vol. 9, No.1, 2018 \\ EDUCATION AND ECONOMIC GROWTH: ANALYSIS AND IMPLICATIONS
}

\section{Adony Ordoñez Espinal}

Universidad Nacional Autónoma de Honduras (UNAH)

Christian Martínez, Santos

Universidad Nacional Autónoma de Honduras (UNAH)

(1) Pedro Zuniga Figueroa

Universidad Nacional Autónoma de Honduras (UNAH)

Carrera de Economía

Ciudad Universitaria, edificio C1, segundo piso, Tel./ Cel. (504) 9765-6636

pedroxzuniga@hotmail.com

Víctor Ordoñez Portillo

Universidad Nacional Autónoma de Honduras (UNAH)

DOI: http://dx.doi.org/10.5377/eya.v9i1.6654

\begin{abstract}
One of the main causes of economic

development is undoubtedly human

capital, the purpose of this document is to present the link that exists between education and economic growth and its different nuances, going from an individual perspective to a macroeconomic perspective.
\end{abstract} Expenditure, Economic Growth.

(1) Corresponding author 


\section{EDUCACIÓN Y CRECIMIENTO ECONÓMICO: ANÁLISIS E IMPLICANCIAS}

Revista Economía y Administración, Vol. 9, No.1, 2018

\section{INTRODUCCIÓN}

La educación es un pilar fundamental para desarrollo de los países, tiene implicancias a nivel individual por el lado de los ingresos y a nivel global ya que contribuye a un mayor progreso para los países en términos de crecimiento económico y cohesión social. La presente investigación tiene como finalidad describir los principales aportes en torno a la discusión teórica y empírica de la educación como motor del crecimiento económico. En lo referente a la participación del sector público en la educación, resulta importante analizar la proporción de recursos que el estado destina a este rubro, y como estos se distribuyen en las diferentes partidas (sueldos y salarios, materiales, infraestructura, transferencias); por otro lado, se compara a la región centroamericana en su nivel de gasto en educación como porcentaje del presupuesto y del PIB. Desde una perspectiva microeconómica se desarrolla el análisis de la educación y el desarrollo económico en cuanto a la rentabilidad que obtiene el individuo al destinar recursos para adquirir mayores cualificaciones y como los beneficios derivados de esa inversión impactan sobre su calidad de vida.

\section{TEORÍA/CÁLCULOS}

Educación y Crecimiento Económico

Desde los inicios de la ciencia económica se valora la relación que existe entre la habilidad de los trabajadores y el incremento en la producción, es decir, de la educación y el crecimiento, según Smith (1794), la división del trabajo otorga muchas ventajas al crecimiento de la producción, ya que por un lado permite ahorro de tiempo, a la vez que mayor destreza en la actividad favorece a aumentos en la cantidad que es capaz de producirse dado un nivel de recursos disponibles.

Sin embargo, no es hasta la mitad del siglo XX que varios economistas demostraron la importancia del factor educativo en el desarrollo de los países, también se encargaron de incluir al capital humano en los modelos de crecimiento económico, los aportes más importantes son:

- Los individuos aumentan su productividad a través del aprendizaje y perfeccionamiento de 


\section{EDUCACIÓN Y CRECIMIENTO ECONÓMICO: ANÁLISIS E IMPLICANCIAS}

Revista Economía y Administración, Vol. 9, No.1, 2018

nuevas habilidades, esto provoca un incremento en sus ingresos, sin embargo, este incremento en la productividad tiene un costo, el cual engloba por un lado el tiempo de esfuerzo por parte de los estudiantes, y por otro lado, el costo de la enseñanza y de los materiales didácticos; las diferencias en los ingresos de los individuos son producto de las diferencias en la tenencia de capital físico, tecnológico y conocimiento o habilidades. (Becker, 1975).

- El capital humano ayuda a explicar la porción residual del crecimiento económico, que no es explicada por el incremento en el capital físico; también el capital humano explica que los ingresos suelen elevarse de forma más pronunciada para las personas con mayor cualificación. (Schultz, 1961).

- Hay dos tipos de capital, por un lado, el capital físico que se utiliza en la producción en función de una determinada capacidad instalada y por otro lado el capital humano que incrementa la productividad de la mano de obra; respecto al ingreso del trabajador, este incrementara en función del país en que se encuentre, por lo tanto, este incrementará de acuerdo a la riqueza del país en que se encuentre, entonces si existe movilidad laboral, los trabajadores se movilizarán hacia los países con mayor nivel de renta. (Lucas, 1988)

- Existe una relación positiva entre el acervo de capital humano de una nación y el crecimiento económico, puesto que entre mayor sea su capital humano el crecimiento económico será más acelerado; también sugiere que los bajos niveles de capital humano explican porque los países de menor desarrollo relativo no tienen el mismo ritmo de los países desarrollados. (Romer, 1990).

- Cuanto mayor sea la cantidad de capital humano por persona, mayores serán las tasas de inversión en capital físico y humano lo cual tiene como efecto un crecimiento del PIB per-cápita; también propone que entre mayor es la ratio 


\section{EDUCACIÓN Y CRECIMIENTO ECONÓMICO: ANÁLISIS E IMPLICANCIAS}

Revista Economía y Administración, Vol. 9, No.1, 2018

maestro-alumno menor es la calidad

educativa. (Barro, 1991).

- Familias con pocos miembros tienden a invertir más en capital humano que las familias de numerosos miembros; por lo tanto, una mayor tasa de fertilidad desalienta la inversión en capital humano. (Becker, Murphy y Tamura, 1990).

En base a lo expuesto anteriormente, resulta indudable que si un país desea tener tasas de crecimiento económico más altas debe invertir en la educación de su población, puesto que esto es un determinante a largo plazo del nivel de desarrollo y bienestar de los países.

Gasto en Educación

\section{La Organización de las Naciones}

Unidas por medio de la Declaración

\section{Universal de Derechos Humanos}

(2006), establece que toda persona tiene derecho a la educación y a la vez que esta debe ser gratuita al menos en la instrucción elemental y fundamental, esto presenta un deber a las naciones para brindar la cobertura y calidad necesarias para el desarrollo de las capacidades cognitivas de los ciudadanos, pero más que un deber se ha convertido en una necesidad ya que está demostrado el papel que juega la educación en el crecimiento de las naciones no solo en el aspecto económico sino que también social y cultural. (art. 26).

La educación no es un bien público puro, puesto que su costo marginal es mayor a cero; además, la justificación del apoyo público a la educación parte del hecho de que la calidad de la educación no debe depender del ingreso de los jefes de hogar. (Stiglitz, 2003).

La distribución del gasto público en educación también es importante, puesto que esta puede estar destinada a diferentes estratos, por ejemplo, educación primaria, secundaria y superior; sin embargo, es ineludible el hecho de que para brindar servicios de educación terciaria se debe garantizar la educación básica (primaria y secundaria), es por ello por lo que en muchos países subdesarrollados el gasto en educación suele ir destinado 


\section{EDUCACIÓN Y CRECIMIENTO ECONÓMICO: ANÁLISIS E IMPLICANCIAS}

Revista Economía y Administración, Vol. 9, No.1, 2018

principalmente a este segmento. (Moreno y Ruiz, 2009).

Según Zoido (2008), las ganancias derivadas de la educación a nivel agregado considera factores más allá de lo académico, por ejemplo la cohesión social, además de esto el gasto público en educación cobra mayor importancia cuando lo asociamos a un contexto o mundo globalizado y competitivo que está en constante cambio, pero cuando hablamos de gasto público en educación no necesariamente el gastar elevados montos de dinero significa mayor calidad educativa, por ejemplo, los sistemas educativos en Lituania y China dedican una cantidad similar a la de los sistemas latinoamericanos, pero los primeros obtienen mejores resultados en los exámenes internacionales estandarizados.

Rentabilidad de la Educación

La educación más que un bien de consumo es considerado una inversión, y como toda inversión debe afrontar costos para obtener beneficios, entonces se puede tratar a la inversión en educación como un proyecto en el cual la tasa de retorno es una medida de la rentabilidad de este, resultado de la comparación de costos y beneficios, directos e indirectos; Algunos de los aspectos que afectan negativamente la rentabilidad de la educación son la repitencia, el ausentismo y la deserción. (Cohen, Schiefelbein, Wolff y Schiefelbein, 2000).

Figura No. 1 - Beneficios y Costos de la Educación Superior

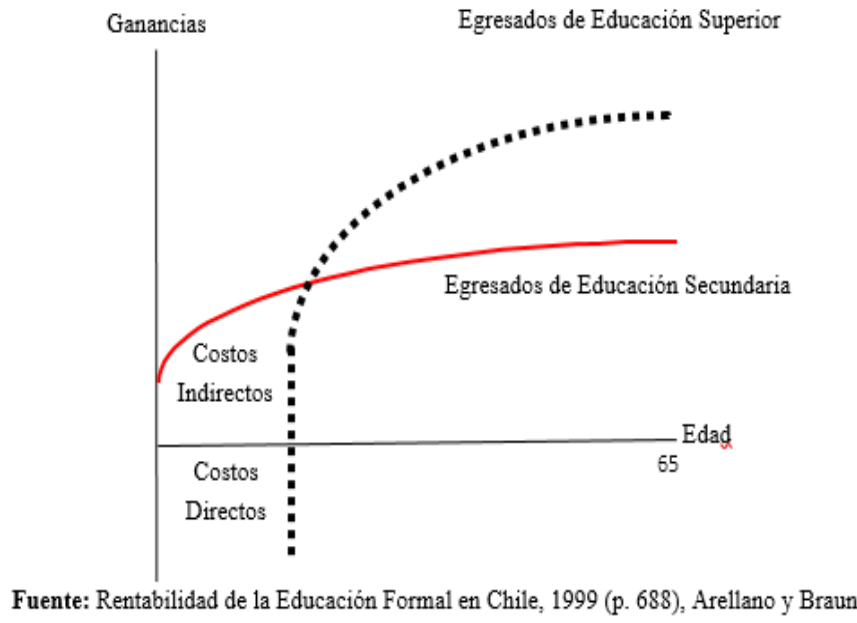

En la Figura No. 1, se presentan las ganancias y costos de la educación superior, los cuales pueden ser directos (desembolsos en materiales, libros, etc.) e indirectos (el costo de oportunidad de trabajar durante se estudia la carrera universitaria), la línea roja muestra los beneficios en caso de no optar por un título de educación superior, mientras que la 


\section{EDUCACIÓN Y CRECIMIENTO ECONÓMICO: ANÁLISIS E IMPLICANCIAS}

Revista Economía y Administración, Vol. 9, No.1, 2018

línea punteada muestra los costos y los

beneficios de obtener un título

universitario, siendo esta la opción más

rentable a simple vista para el

individuo ya que los años posteriores a

la obtención del título recupera la

inversión (costos) y obtiene mayores

ingresos. (Arellano y Braun, 1999).

Esto está relacionado al ciclo de vida en el cual el individuo enfrenta una etapa donde sus ingresos son negativos (estudio), una en la que son positivos (inserción en el mercado laboral, comienza a ahorrar), posteriormente alcanza un máximo de ingreso y a partir de ese punto su riqueza comienza a decrecer. (Modigliani, 1986).

\section{METODOLOGÍA}

La investigación tiene como alcance un enfoque explicativo y un diseño no experimental de corte transversal; con la intención de cuantificar y comprobar algunas de las teorías anteriormente descritas, se planteó lo siguiente:

- Con el fin de conocer el impacto de la educación en el crecimiento económico, se realizó un modelo econométrico basado en el trabajo realizado por Kovacs (2007), en cual se utiliza como variable dependiente el PIB per cápita, mientras que como variables independientes (que explican las variaciones del PIB per cápita) al gasto público en educación respecto al PIB, la tasa de analfabetismo, el ratio alumno-maestro y una constante; en lo que respecta al modelo elaborado en la presente investigación se tomaron 26 observaciones para su desarrollo.

- En cuanto al análisis del gasto en educación para la región centroamericana, se utilizaron datos del presupuesto general de ingresos y egresos para cada país, a la vez que se determinó el porcentaje de dicho presupuesto que se destina en educación (en moneda nacional y extranjera), para completar el análisis del gasto se tomó PIB nominal para así obtener la relación entre dicha variable y el gasto público en educación.

- Para determinar la rentabilidad de la educación se llevó a cabo el cruce de tres variables, edad, ingreso y nivel educativo utilizando como fuente la Encuesta Permanente de 


\section{EDUCACIÓN Y CRECIMIENTO ECONÓMICO: ANÁLISIS E IMPLICANCIAS}

Revista Economía y Administración, Vol. 9, No.1, 2018

Hogares de Propósitos Múltiples (EPHPM) del año 2013, esto con la intención de relacionar el ingreso del individuo con su nivel educativo y la diferencia de ingresos que existe entre las personas dependiendo de su grado académico, por último, describir el comportamiento de los ingresos respecto a la edad de estos.

\section{RESULTADOS}

Educación y Crecimiento Económico

Modelo de Series de Tiempo

El modelo utilizado en la presente investigación es de largo plazo y parte de la siguiente ecuación:

Ec. (1)

$$
\begin{gathered}
\log Y_{P}=C+\beta_{0} \operatorname{LogGP_{E}}-\beta_{1} \log T_{A} \\
+U_{i}
\end{gathered}
$$

Tal y como se muestra en la ecuación [Ec. (1)], $\mathbf{Y}_{\mathbf{P}}$ representa el ingreso per cápita, $\mathbf{G P}_{\mathbf{E}}$ es la ratio del presupuesto en educación respecto al PIB, $\mathbf{T}_{\mathbf{A}}$ es la tasa de analfabetismo, $\mathbf{C}$ es una constante, $\boldsymbol{\beta}_{\mathbf{0}}$ y $\boldsymbol{\beta}_{\mathbf{1}}$ son los coeficientes de las variables independientes y $\mathbf{U}_{\mathbf{i}}$ simboliza el error estocástico.
Tabla No. 1 - Resultados del Modelo Econométrico.

\begin{tabular}{|c|c|c|c|}
\hline Variable & Coeficiente & T-Estadistico & Probabilidad \\
\hline$C$ & 9.104154 & 16.94855 & 0.0000 \\
\hline $\log (G P e)$ & 0.724140 & 6.920951 & 0.0000 \\
\hline $\log (T a)$ & -1.795753 & -12.09374 & 0.0000 \\
\hline $\mathrm{R}^{2}$ & 0.972581 & - & - \\
\hline R Ajustado & 0.970196 & - & - \\
\hline
\end{tabular}

Fuente: Elaboración propia con datos del BCH y SEFIN.

Como se puede observar en la Tabla No. 1, las variables gasto público en educación y tasa analfabetismo son estadísticamente significativas en el largo plazo, ya que superan la prueba " $T$ " de significancia, por lo cual se puede decir que explican el comportamiento del PIB per-cápita en el periodo de estudio.

El coeficiente de determinación o $\mathrm{R}^{2}$ es de 0.97 , lo que quiere decir que las variables independientes explican en un $97 \%$ a la variable dependiente.

Respecto a los signos, se puede observar que hay una relación directa entre la ratio del gasto público en educación y el PIB per-cápita lo cual es sustentado por la teoría, caso distinto al de la tasa de analfabetismo que presenta una relación inversa con el PIB per-cápita lo cual quiere decir que una disminución en la tasa de 


\section{EDUCACIÓN Y CRECIMIENTO ECONÓMICO: ANÁLISIS E IMPLICANCIAS}

Revista Economía y Administración, Vol. 9, No.1, 2018

analfabetismo corresponde a un

incremento en la producción.

\section{Gasto en Educación}

Gasto Público en Educación: Honduras

Tabla No. 2 Gasto Público en Educación Desagregado (2015).

\begin{tabular}{|c|c|c|}
\hline Categoria & Orientación del Gasto & $\begin{array}{c}\text { \% del Gasto Total } \\
\text { Aprobado }\end{array}$ \\
\hline 1 & Servicios Personales & $79,23 \%$ \\
\hline 2 & $\begin{array}{c}\text { Servicios No Personales, Materiales y } \\
\text { Suministros y Bienes Capitalizables }\end{array}$ & $1,29 \%$ \\
\hline 3 & \multicolumn{2}{|c|}{ Transferencias } \\
\hline \multicolumn{2}{|c|}{ Fuente: Elaboración propia con datos de la Secretaria de Educación de Honduras. }
\end{tabular}

En la Tabla No. 2 se presenta el grado de participación de las principales partidas que conforman el presupuesto en educación, en las cuales figuran los servicios personales $(79.23 \%)$, seguidos de las transferencias $(19.48 \%)$, y en último lugar los servicios no personales, materiales y bienes capitalizables (1.29\%), confirmando de este modo que el mayor monto de dinero del presupuesto en educación es destinado al pago de sueldos y salarios, quedando muy poco para la inversión en infraestructura y materiales.

\section{Gasto Público en Educación:}

\section{Centroamérica}

El papel del Estado en la educación es fundamental, es pues por eso que los gobiernos desembolsan grandes cantidades de dinero en esta actividad, por esa razón es necesario hacer un análisis desagregado y comparativo a nivel regional (Centroamérica), que permita entender la magnitud del gasto en Honduras como en los demás países.

Figura No. 2 Comparativa del Gasto Público en Educación Centroamérica (2015).

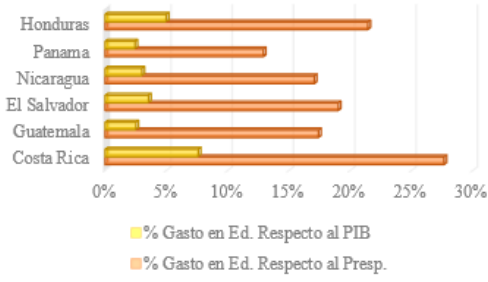

Fuente: Elaboración propia con datos de las Instituciones de Hacienda y Finanzas Publicas de Centroamérica.|

Tal y como se observa en la Figura No.

2 , el gasto en educación realizado por el gobierno de Honduras para el año 2015 fue de aproximadamente 22 mil millones de lempiras, lo que representa un $21 \%$ del presupuesto nacional y un 5\% del PIB, lo que señala una nada despreciable cantidad de dinero destinada a tan importante rubro. Por otro lado Costa Rica es el país que más gasta en educación en la región centroamericana, representando un 


\section{EDUCACIÓN Y CRECIMIENTO ECONÓMICO: ANÁLISIS E IMPLICANCIAS}

Revista Economía y Administración, Vol. 9, No.1, 2018

$27 \%$ del presupuesto y un $7.6 \%$ del

PIB, Guatemala gasta alrededor del

$17.4 \%$ de su presupuesto en educación

$\mathrm{y}$ un $2.5 \%$ del PIB, mientras que $\mathrm{El}$

Salvador destina un $19 \%$ de su

presupuesto en educación, siendo como

porcentaje del PIB un $3.5 \%$, Nicaragua

por su parte gasta $17 \%$ de su

presupuesto en educación, lo que representa un 3\% de su PIB, y Panamá

es el país que menos invierte en educación siendo el gasto público solamente un $12 \%$ del presupuesto y un $2.4 \%$ del PIB.

Tabla No. 3 Gasto Público en Educación en Moneda Extranjera en 2015 (Dólares)

\begin{tabular}{|c|c|}
\hline País & Gasto en Ed. $\$$ \\
\hline Costa Rica & $\$ 4,117,112,848.86$ \\
\hline Guatemala & $\$ 1,607,266,764.58$ \\
\hline El Salvador & $\$ 917,700,000.00$ \\
\hline Nicaragua & $\$ 382,214,782.28$ \\
\hline Panamá & $\$ 1,272,396,000.00$ \\
\hline Honduras & $\$ 1,015,441,927.73$ \\
\hline
\end{tabular}

Fuente: Elaboración propia con datos del SECMCA e Instituciones de Hacienda Publica de Centroamérica.

La Tabla No. 3 refleja que en moneda extranjera (dólares) y basándose en el tipo de cambio promedio anual del 2015, Costa Rica destino 3,040 millones de dólares en educación, mientras que Honduras empleó 1,015 millones de dólares, el país centroamericano que menos invirtió en educación en el 2015 fue Nicaragua siendo su gasto en educación 382 millones de dólares; sin embargo y antes de apresurarse a tomar conclusiones, cabe recalcar que un mayor nivel de gasto no se traduce en mejor rendimiento tal y como se comentó en el marco de teorías, hay que analizar los resultados en base a indicadores educativos, para observar si la relación gasto-rendimiento es válida para este análisis.

Tabla No. 4 Comparativa Gasto Público en Educación Centroamérica (2000)

\begin{tabular}{|c|c|c|}
\hline \multirow{4}{*}{ País } & Gasto & Gasto \\
& Publico en & Publico \\
& Ed. cómo & en Ed. \\
& $\%$ & cómo \% \\
& Presupuest & PIB \\
\hline
\end{tabular}




\section{EDUCACIÓN Y CRECIMIENTO ECONÓMICO: ANÁLISIS E IMPLICANCIAS}

Revista Economía y Administración, Vol. 9, No.1, 2018

\begin{tabular}{|c|c|c|}
\hline $\begin{array}{c}\text { Costa } \\
\text { Rica }\end{array}$ & $21.36 \%$ & $4.67 \%$ \\
\hline $\begin{array}{c}\text { Guatemal } \\
\text { a }\end{array}$ & $12.07 \%$ & $2.02 \%$ \\
\hline $\begin{array}{c}\text { El } \\
\text { Salvador }\end{array}$ & $17.47 \%$ & $2.94 \%$ \\
\hline Honduras & $21.31 \%$ & $4.51 \%$ \\
\hline $\begin{array}{c}\text { Nicaragu } \\
\text { a }\end{array}$ & $18.64 \%$ & $3.00 \%$ \\
\hline
\end{tabular}

Fuente: Elaboración propia con datos de las Instituciones de Hacienda y Finanzas Publicas de Centroamérica.

Es necesario hacer una retrospectiva para determinar si el gasto público en educación de los países en Centroamérica ha tenido algún efecto en los indicadores de rendimiento educativo, resultado de ello la Tabla No. 4 se presenta para el año 2000 la situación del gasto en educación; Costa Rica desembolsó en educación 697 millones de dólares (4.7\% del PIB) siendo el país que más invirtió en este rubro, mientras que Honduras destinó aproximadamente 324 millones de dólares (4.5\% del PIB), Guatemala 346 millones de dólares (2\% del PIB), Nicaragua 153 millones de dólares (3\% del PIB), El Salvador 386 millones de dólares y Panamá 453 millones de dólares.

Con la finalidad de relacionar el gasto en educación y los resultados educativos se tomó como referencia la tasa de analfabetismo (ya que la alfabetización otorga a la población competencias necesarias para dar continuidad al proceso de formación educativo, puesto que favorece a un aumento en el potencial para perfeccionar el intelecto, lo que contribuye al desarrollo económico y sociocultural de la sociedad), tal y como se muestra en la Figura 3, Costa Rica tenía una tasa de analfabetismo de 4.4\% en el año 2000 y logró disminuirla a $2.2 \%$ en el 2015, mientras que Guatemala reportó una tasa de analfabetismo de $31.67 \%$ en el año 2000 siendo el país con mayores problemas de analfabetismo de la región centroamericana, para el 2015 logró reducir su tasa de analfabetismo a $13.09 \%$, por otro lado El Salvador reportó una tasa de analfabetismo de $19 \%$ (en el 2000) y de 12\% (en 2015), Nicaragua de $23 \%$ (en el 2000) y $18 \%$ (en 2015) mientras que Honduras en el 2000 tuvo una tasa de analfabetismo de $19 \%$ y logró reducirla a $12.1 \%$ en el 2015.

Figura No. 3 Tasa de Analfabetismo en Centroamérica (Personas Mayores de 15 Años) 


\section{EDUCACIÓN Y CRECIMIENTO ECONÓMICO: ANÁLISIS E IMPLICANCIAS}

Revista Economía y Administración, Vol. 9, No.1, 2018

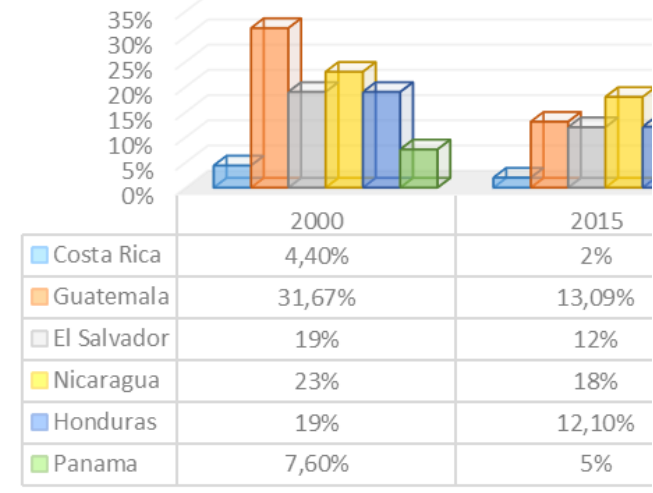

Fuente: Elaboración propia con datos del Banco Mundial e Institutos de Estadística de Centroamérica.

En base a esos resultados y revisando los montos de presupuesto destinado en los países centroamericanos en educación se puede concluir claramente que Costa Rica que es el país que más invierte en educación es a la vez el que mejores resultados ha obtenido, Guatemala que solo invierte en educación $2.52 \%$ del PIB logró reducir en $18 \%$ su tasa de analfabetismo en 15 años, mientras que Honduras redujo el analfabetismo en $7 \%$ en el mismo periodo pero invirtiendo cerca del $5 \%$ del PIB en educación, a simple vista parece que Guatemala tiene mejores resultados con un menor gasto como porcentaje del PIB y es cierto, por ello es necesario revisar los montos en moneda extranjera (para una comparación homogénea), de esta forma se puede observar que Guatemala destina una mayor cantidad de dinero en educación que Honduras, aunque como proporción represente un valor menor de su PIB.

\section{Rentabilidad de la Educación}

Se realizó un cruce de variables para conocer el impacto del nivel educativo en los ingresos del individuo, también se incluyó la variable edad para analizar la evolución del ingreso per cápita promedio de los hogares, para efectos de la investigación cabe destacar que se segmentaron los datos por dominio y solo se consideró el área urbana debido a la estructura del mercado laboral.

Figura No. 4 Relación Ingreso-EdadNivel Educativo (2013) 


\section{EDUCACIÓN Y CRECIMIENTO ECONÓMICO: ANÁLISIS E IMPLICANCIAS}

Revista Economía y Administración, Vol. 9, No.1, 2018

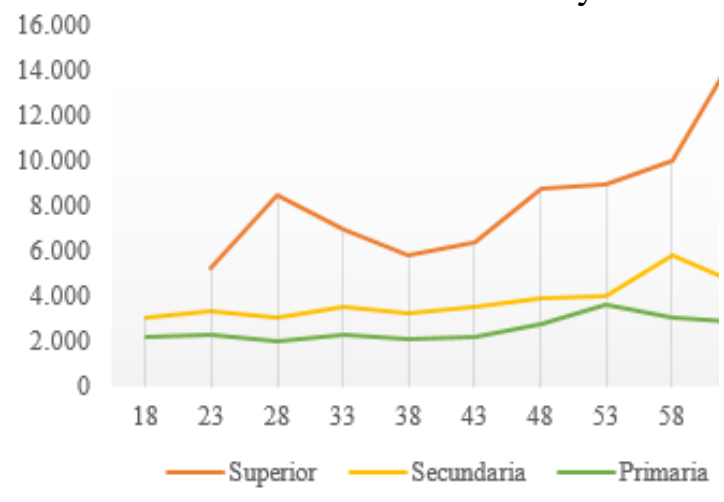

Fuente: Elaboración Propia con datos del INE.

Se pueden observar en la Figura No. 4, las diferencias en los ingresos de los individuos de educación superior y educación media, y en menor cuantía los individuos de educación media y educación primaria, por ejemplo, los individuos de 23 a 27 años, con educación superior ganan $58 \%$ más que los poseen educación secundaria, en contraste los individuos que cuentan solo con educación media para el rango de edad anteriormente descrito ganan $46 \%$ más que los que poseen educación primaria, lo que sin lugar a dudas demuestra que los individuos con mayor nivel educativo presentan mayor nivel de ingresos, corroborando así que invertir en educación es rentable. En cuanto a la relación edad-ingreso se puede observar que los individuos experimentan un periodo de ingresos máximos y que posterior a alcanzar este punto los ingresos comienzan a disminuir, los individuos que cuentan con educación primaria encuentran su punto máximo de ingreso a una edad más temprana que los que cuentan con educación secundaria y superior.

\section{CONCLUSIONES}

En base a los resultados obtenidos del modelo econométrico se pudo confirmar la relación que existe entre la educación y el crecimiento económico, siendo la tasa de analfabetismo y el gasto público en educación los indicadores educativos que más impacto tienen (estadísticamente) en el PIB per cápita, por otro lado es importante mencionar que Honduras no cuenta con una base de estadísticas educativas basta, lo que no permite incluir otras variables que en la teoría también suelen ser utilizadas, además la relación entre las variables modelizadas corresponde a la teoría, donde una disminución en la tasa de analfabetismo favorece a incrementos en la producción, lo cual en concordancia con las teorías expuestas, donde un mayor nivel educativo (capital humano), corresponde a incrementos en la producción, por otro lado aumentos 


\section{EDUCACIÓN Y CRECIMIENTO ECONÓMICO: ANÁLISIS E IMPLICANCIAS}

Revista Economía y Administración, Vol. 9, No.1, 2018

en el gasto público en educación

favorece a incrementos en el

crecimiento económico per-cápita.

El papel del estado en la provisión de servicios educativos siempre ha sido relevante, y reflejo de esto es el presupuesto que los países destinan en educación, ya que una población educada por un lado permite aumentos en la productividad y por otro lado da lugar a que se suscite mayor cohesión social; en la región centroamericana el país que más destina recursos a la educación es Costa Rica, seguido de Guatemala y Panamá, esto en términos de dólares, como porcentaje del PIB es Costa Rica (7.6\%) quien mayor proporción destina en recursos, seguido de Honduras (5\%), sin embargo, no solo se trata de analizar el gasto en educación en términos de magnitud sino también en objeto de gasto, en el caso de Honduras la mayoría de recursos presupuestarios en educación se destinan a servicios personales (pago de sueldos y salarios), dejando un pequeño porcentaje en inversión en infraestructura y materiales .

A través de la Encuesta Permanente de Hogares de Propósitos Múltiples se logró determinar que en la relación edad-ingreso, la renta independientemente del nivel educativo es creciente hasta alcanzar un punto máximo y que a partir de ese punto esta comienza a disminuir (en secundaria no se pudo mostrar la relación); además que entre mayor es el nivel educativo de los individuos, mejores posibilidades tiene de aspirar a mayores ingresos, lo anteriormente expuesto permite concluir que invertir en educación sobre todo a nivel superior es algo necesario en la actualidad, pero para ello primero se debe de garantizar una cobertura y calidad adecuada en la educación primaria y secundaria, un reto que han y continúan enfrentando los países latinoamericanos por muchos años.

\section{REFERENCIAS}

ARELLANO, S. and BRAUN, L., 1999. Rentabilidad de la educación formal en Chile. Cuadernos de Economic, 36(107), 685-724.

BARRO, R., 1991. Economic growth in a cross section of countries. The Quarterly Journal of Economics, 106(2), 407-443. 
EDUCACIÓN Y CRECIMIENTO ECONÓMICO: ANÁLISIS E IMPLICANCIAS

Revista Economía y Administración, Vol. 9, No.1, 2018

BECKER, G., 1975. Human capital: a theoretical and empirical analysis, with special reference to education $\left(2^{\mathrm{a}}\right.$ ed.). New York: National Bureau of Economic Research.

BECKER, G., MURPHY, K. and TAMURA, R., 1990. Human capital, fertility, and economic growth. Journal of Political Economy, 98(5), 12-37.

COHEN, E., SCHIEFELBEIN, E., WOLFF, L. and SCHIEFELBEIN, P., 2000. ¿Hacia dónde va el gasto público en educación? logros y desafíos. Santiago de Chile: Naciones Unidas.

KOVACS, M.L., 2007. Educación y crecimiento económico (tesis de pregrado). Universidad Cattolica de Argentina, Argentina.

LUCAS, R., 1988. On the mechanics of economic development. Journal of monetary economics, 22(1), 3-42.
MODIGLIANI, F., 1986. Life cycle, individual thrift, and the wealth of nations. The American Economic Review, 76(3), 297-313.

NACIONES UNIDAS., 2006. Declaración Universal de los Derechos Humanos. Los principales tratados Internacionales de derechos humanos. New York y Ginebra.

ROMER, P., 1990. Endogenous technological change. Journal of Political Economy, 98(5), 71-102.

SCHULTZ, T., 1961. Investment in human capital. The American Economic Review, 51(1), 1-17.

SMITH, A., 1794. Investigación de la naturaleza y causes de la riqueza de las naciones (Vol. 1). España: En la Oficina de la Viuda e Hijos de Santander. 


\section{EDUCACIÓN Y CRECIMIENTO ECONÓMICO: ANÁLISIS E IMPLICANCIAS}

Revista Economía y Administración, Vol. 9, No.1, 2018

STIGLITZ, J.E., 2003. La economía del

sector público ( $3^{\mathrm{a}}$ ed.). España: Antoni

Bosch.

ZOIDO, P., 2008. El gasto público en

educación en América Latina: ¿da

resultado? Percepciones, (80). 


\section{EDUCACIÓN Y CRECIMIENTO ECONÓMICO: ANÁLISIS E IMPLICANCIAS}

Revista Economía y Administración, Vol. 9, No.1, 2018 